This line of thought raises questions as to the effect of the enormous mass of positive luminous vapours participating in the solar rotation. Will it give rise to magnetic effects? Perhaps not if ether is dragged with the sum; at any rate, a charged body rotating with the earth seems to produce no magnetic field. If, however, there are solar atmospheric currents on a large scale magnetic effects may be expected. If the sun is thus magnetised it would act inductively on the earth, and the magnetism of the earth might be thus accounted for. The sun's north pole should be positive to account for the negative polarity of the earth's geographic pole, and it is interesting to note that such would be the case if the sun's polarity is due to positively charged vapour rotating with it.

It would likewise follow that the moon is an induced magnet. It might be worth while for someone to consider the effects upon perturbations of earth and noon of the force moments due to the non-coincidence of the polar and magnetic axes.

If the supposed positive charges in the chromosphere arise from the projection of electrons into space, the accumulation of the latter in outer regions would produce with the positive atmosphere an enormous electrical double layer, with a radial field which would restrain further travel of the electrons and perhaps cause the tremendous outbursts of luminous positively charged vapour shown in prominences. Magnetic effects in these prominences should be looked for.

Very light ions (perhaps of some lighter element than any we know) might be drawn from the sun by the electric field, and their subsequent neutralisation by electrons may give rise to coronal line radiation. These encounters, always taking place in radial lines, may give rise to partially polarised radiation, but it must be confessed that it seems difficult to reconcile the effects which would probably follow with the observed direction of vibration in coronal light.

University of California, September I4.

\section{Memory in the Germ-plasm.}

IT has recently been suggested that acquired characters are transmitted by a kind of memory in the germ-plasm. If this suggestion were adopted, would it not enable us to explain the non-transmission of mutilations?

If there be such a connection between the somatic-cells and the germ-cells as this new theory presupposes, that connection must be constant. We must suppose the germcells to be a kind of registry in which all the events of the somatic life are recorded. Many of the records would be evanescent, just as many of the records in consciousness are evanescent, but important somatic changes would (by the accumulation of impressions) produce perduring records, and these would be the biological ground of the transmission of those changes to the new generation.

Now consider what happens in mutilation. A lamb's tail is shortened-what is the result in germ-memory? Merely the record of a momentary cut. Why should this be transmitted? There is already in the germ-memory the record of an undiminished tail-a record produced by thousands of impressions accumulated through every moment of the animal's earlier life. Naturally, this record will be prepotent over the record of a momentary event.

We must remember, too, that the nerves and muscles of a stump often strangely preserve for a long time what may almost be called a recollection of the amputated part. If the lost limb thus perpetuate itself (so to speak) in consciousness, it seems probable, ex hypothesi, that it similarly perpetuates itself in germ-memory. Moreover, a man who has lost a leg constantly tries (consciously or subconsciously) to act as though he still had it. This, again, one may suppose, would tend to perpetuate the germ-record of the lost member.

It seems, then, that, in a case of mutilation, the record in germ-memory of the momentary act of mutilation would be an evanescent record, and the germ-record, as a whole, would continue to be the record of an unmutilated body. Does not this help us to understand the non-transmission of mutilations?

Hakluyt Egerton.

\section{Models of Plane and Spherical Waves.}

IT is very easy to form a mental picture of the displacements in an isotropic elastic solid transmitting a plane transverse wave. Alternate planes of constant phase are sheared relatively to one another, as explained in Schuster's "Optics," § I2. If this be a correct representation of the process of transmission, it should be possible to apply a similar method to the alternate spherical shells in the transmission of a spherical wave. The shears must obviously possess symmetry about the point centre of the disturbance, since the waves are transmitted uniformly in every direction. This appears to me to be impossible; but if such shears are impossible in a spherical wave it is absurd to apply them to a particular case of the spherical wave, viz. a plane wave. Will some kind friend please explain?

J. J. D.

THE following explanation may be helpful if I understand the difficulty aright.

Imagine a number of concentric spherical shells like the layers of an onion. Imagine the inside shell rotated about a diameter through a small angle, and imagine this displacement taken up in succession by the next shell, the next but one, and so on. Then we have a disturbance radiating outwards from the centre, but the wave motion is not symmetrical about the centre. If, however, we go to a long distance from the inside sphere along a perpendicular diameter we get practically plane waves.

But these plane waves are in no way the limiting case of spherical waves symmetrical about a point, for the vibrations take place in a particular direction (in other words, they are plane polarised). It is impossible to have shears in plane waves which possess the symmetry referred to by your correspondent. A pack of cards can be sheared parallel to its longer or shorter side or to its diagonal, but it cannot be sheared at the same time equally in all directions. There is no difference between plane and spherical waves in this respect. Light spreading out uniformly from a source is a very different thing from these simple plane and spherical waves. It represents a jumble of waves sent out from a large number of molecules, and these molecules are not only moving about and changing their positions, but are themselves rotating. The radiations they emit are probably different from the motions of the spherical shells described above, but these ao by way of illustration.

G. H. B

\section{The Pendulation Theory.}

In NATURE of April 2, 1908, the reviewer of my "Pendulationstheorie," Mr. R. L., directed attention to the map which shows the distribution of the ichthyosaurs, because I have left out the African ones. He therefore thinks it difficult to commit himself to an opinion on the theory.

Those ichthyosaurs in Africa (not yet marked in the edition of Zittel's "Palæontology" I have used) are not in the least an argument against my theory, but give me another most striking proof of it. I had shown that the ichthyosaurs had taken their origin under the pendulation circle, and had wandered from there on the usual line south-eastward to New Zealand. For they have been found under the pendulation circle from Spitsbergen to Sicily, all the others on a line from Europe to New Zealand.

Their having been found in Africa only enlarges their extension along the pendulation circle, and so confirms my opinion that the ichthyosaurs have here taken their origin.

Another instance, quite recently discovered, offers the fluviatile medusa Limnocodium. Until recently it was only known in aquariums; it was first found in England, and described by Lankester. Its occurrence together with Victoria regia proves that its origin is in the tropical parts of America. Some time ago Prof. Oka described a second species from the upper Yang-tse-kiang. That is just the same discontinuous occurrence which I have pointed out, for instance, for the alligators, and which I have made use of for the pendulation theory.

Leipzig, Gautzsch, August 2 r. 